\title{
PERCEPCION DE VIOLENCIA DE LOS PACIENTES HOSPITALIZAD OS EN LOS SERVICIOS DE MEDICINA Y CIRUGIA DE UN HOSPITAL PUBLICO ${ }^{1}$
}

\author{
PERCEPTION OF VIOLENCE BY PATIENTS HOSPITALIZED IN \\ THE MEDICINE AND SURGERY SERVICES OF A PUBLIC HOSPITAL
}

MÓNICA DEL CARMEN BURGOS MORENO* y TATIANA MARÍA PARAVIC KLIJN**

\begin{abstract}
RESUMEN
Estudio de tipo descriptivo, comparativo y correlacional, cuyo objetivo general fue conocer la percepción de violencia de los pacientes hospitalizados en los servicios de medicina y cirugía de un hospital público y las variables que se encuentran relacionadas. Esta investigación se basó en el Modelo Interactivo de Violencia Laboral de Chapell y Di Martino, aplicado al ambiente hospitalario.

La muestra estuvo conformada por 174 pacientes, 70 del servicio de medicina y 104 de cirugía. Algunos de los resultados fueron: un $35,7 \%$ de los pacientes de M edicina y un $42,3 \%$ de los pacientes de Cirugía perciben violencia durantela atención hospitalaria. Estas percepciones de violencia corresponden a los pacientes más jóvenes con mayor nivel educacional y que evalúan mal la atención recibida. Las conductas violentas se presentaron con mayor frecuencia durante los turnos de noche, entre las que se destacaron la "falta de atención" y el "trato frío" de parte del personal de salud. Los usuarios identificaron que el estrés por exceso de trabajo delos funcionarios de la salud constituye el factor predisponente más importante de conductas violentas.
\end{abstract}

Palabras claves: Violencia hospitalaria, percepción de violencia, paciente hospitalizados en los servicios de medicina y cirugía.

\begin{abstract}
This is a descriptive, comparative and co-relational study. Its general objective consisted in finding out about hospitalized patients' perception of violence and its related variablesin the medicineand surgery wards of a public hospital. Thestudy is based on Chapell and Di Martino's Interactive M odel of work-related violence applied to thehospital environment.

The samplewas made up of 174 patients; 70 from the medicineward and 104 from the surgery service. Some of the findings were the following: $35.7 \%$ of the patients in the medicine service, and $42.3 \%$ of the patients in the surgery ward perceived violence while receiving hospital care. This perception of violence corresponds to younger patients who have a higher educational level. They rate the care received as deficient. Violent behavior was most frequently perceived during night shifts. "Lack of care" and "impersonal treatment" from hospital personnel, were the attitudes most frequently perceived by these patients. They relate excessive work stress imposed on the personnel of the health care system as the main factor accounting for violent behavior.
\end{abstract}

Keywords: Hospital violence, perception of violence, patient hospitalized in the medicine and surgery wards.

Recepcionado: 1.06.2003. Aceptado: 29.09.2003.

\section{INTRODUCCIÓN}

El aumento considerable dela violencia es un fenómeno reconocido ampliamente. Según el
Consejo Internacional de Enfermería (CIE, 1999), este fenómeno afecta a toda la población, atravesando fronteras deedad, raza, condición socioeconómica, sexo y lugar.

'Este artículo es parte de la tesis para optar al grado de M agister en Enfermería.

*Instructora D epartamento de Enfermería, Facultad deM edicina, Universidad de Concepción, Chile. Enfermera de la Unidad deRecuperación Anestésica, Hospital LasHigueras, Talcahuano, Chile. Fono: (56-41) 501387.E-mail:moniburgos@udec.cl

**Profesor Titular del Departamento de Enfermería, Universidad deConcepción, Chile. M agister en Enfermería en Salud Comunitaria. Doctora en Enfermería. Orientadora de tesis. Fono (56-41) 204832. E-mail: tparavic@udec.cl 
Diversos estudios en la temática de la violencia que afecta a los espacios laborales concluyen que precisamente al gunos de estos lugares detrabajo y ocupacionestienen un mayor riesgo de hechos violentos que otros. Se reconoce en estas investigaciones que la violencia en el lugar de trabajo en el sector salud es universal, la que genera un al to grado de estrés, lo que también puede instar a comportamientos violentos, y en las que se evidencia quelas enfermeras y el personal de ambulancias tie nen mayor riesgo a ser víctimas de hechos agresivos, el que especialmente corresponde a violencia de tipo psicológico (CIE, 2002; Sánchez, 2002). Para Kingma (1999), la violencia que afecta a las enfermeras tiene una doble consecuencia, ciertamente negativa, porque afecta por un lado la vida de este profesional y, por el otro, puede afectar el tipo de servicios de salud prestados, es decir, la repercusión se pudiera extender a la calidad de los cuidados de salud otorgados a los pacientes. Así, este complejo fenómeno de la violencia es reconocido también en el ambiente hospitalario, enfocado desde la perspectiva de los trabajadores de la atención de salud. Sin embargo, estudios orientados en este tema desde el punto de vista de los pacientes o usuarios de los servicios de salud, principal foco deatención en este contexto, han sido escasamente abordados.

La condición de hospitalización hace al individuo vulnerablea variados estímulos, no sólo provenientes de la enfermedad que le afecta, sino también del medio ambiente y relaciones que se establecen durante la hospitalización, en el que adopta o pasa a ser incluido en el "status de paciente" (Martínez, 1997). Al respecto, Jiménez (2000) señala que este paciente ve alterado su autoestima y se producen cambios emocionales intensos que requieren de reajustes. Estos cambios, para M oro (1999), repercuten en las necesidades de seguridad y pertenencia, en la que un medio extraño, con una serie de normas y a vecesfalto deprivacidad, potencialos sentimientos de inferioridad, aparecen sentimientos de culpa que aumentan con el tratamiento despersonalizado de los procesos diagnósticos, terapéuticos y asistenciales a los que se ve sometido durante la hospitalización.

Entonces el hospital, junto con ofrecer atención sanitaria, se constituyeen un sistema social complejo y delicado, en el que la multiplicidad de personas, con diferentes roles, conforman una red interactiva que puedeinducir adesarrollar o modificar actitudes (Valenzuela, 1995), las que pueden predisponer a hechos agresivos o violentos

Existen ciertas situaciones que se presentan durante la atención que se brinda al pacientehospitalizado y quetal vez, asociadosa la masificación y complejidad creciente de la atención de salud, no sean analizados con la frecuencia y profundidad que merecen, y que pueden ser considerados manifestaciones de agresión o violencia.

Uno de ellos que pareciera ser inherente a la hospitalización, es la falta de intimidad, de finida como "toda aquella realidad oculta, relativa a un sujeto o grupo determinado que merece reserva". Ésta se ve de al guna manera vulnerada en el paciente hospitalizado, el que se manifiesta desde la invasión no sólo al espacio territorial, reducido a una cama y un ve lador (Vacarezza, 2000), sino también al ser examinado en su unidad por innumerables personas a las que generalmente desconoce y respondiendo preguntas relativas a su historia de vida personal, muchas veces sin una explicación previa del porqué son necesarios estos procedimientos y estas respuestas. Pareciera ser más importante el órgano afectado que la persona enferma, olvidando el respeto a la desnudez y el pudor del otro (Rodríguez, 1999). Esto se ha ido presentando en la medida en que la enfermedad monopoliza la atención einconscientemente se olvida la naturaleza humana de la atención de salud, tornándose ésta fría e impersonal, lo que ha violentado la relación amistosa y de confianza, pasando más bien a un vínculo de tipo despersonalizado (Iceta, 1996; Goic, 2000). Esto estaría asociado a lo que jiménez y cols. (1990) 
Ilaman una relación asimétrica y jerárquica entre los dispensadores de cuidados y los pacientes.

En una relación depoder, por ejemplo, que establecen las enfermeras con los pacientes hospitalizados, provocan una actitud obediente, sumisa y pasiva del usuario frente a las diversas relaciones e interacciones que se presentan. En un estudio realizado por M oris, Riverosy Pucheau (1996), seidentificaron los factores significativos en la percepción de la atención que recibían los pacientes hospitalizados en un hospital público, resultando que los pacientes consideraban importante las relaciones que se establecen con el equipo de salud, identificando como un atributo significativo el trato respetuoso del personal que los atendía.

En el contexto hospitalario el foco deatención es el cuidado del paciente hospitalizado y sus necesidades, lo que implica identificar constantemente los factores internos y externos que están influyendo en la salud de los que están a nuestro cargo, es decir, la atención de enfermería está dirigida al individuo en forma integral, contemplando distintostipos de estímulos y respuestas en este medio, muchas veces desconocido y doloroso para quienes, por distintos motivos, presentan alguna alteración en el estado de salud, de resolución médica o quirúrgica, y que requieren el ingreso a un establecimiento hospitalario.

Los pacientes, receptores de los servicios sanitarios, pueden ser violentadosa través de variadas manifestaciones, que vulneran sus derechos y los que muchas veces no son solamente ignorados por los dispensadores de la atención de salud, sino también por los mismosusuarios. En una atención que ha priorizado la tecnificación y conocimientos teóricos que son elementos importantes en la atención en salud, éstos no son suficientes si se vulneran los derechos a una atención basada en el trato humano, lo quepermite otorgar ciertamente no sólo una mejor atención, sino que un cuidado más digno.

EI CIE (2002) ha adoptado una clara opo- sición en estereferente, condenando todaforma de abuso y de violencia en los contextos hospitalarios, es por esta razón quejunto con la Organización Mundial de la Salud (OM S), Organización Internacional del Trabajo (OIT) y la Internacional deServicios Públicos(PSI), establecen una campaña para erradicar la violencia en el sector salud, que según reconocen influye en los cuidados brindados a los pacientes.

Las escasas investigaciones en este tema desde la perspectiva de los pacientes y, por otro lado, el interés de los autores de realizar un diagnóstico en dos servicios clínicos hospitalarios sobre la violencia en la atención de salud otorgada a sus usuarios, Ilevaron a plantear los siguientes objetivos e hipótesis de investigación:

\section{OBJETIVOS DE INVESTIGACIÓN}

- Determinar la percepción de violencia en la atención de salud desdela perspectiva delos pacientes adultos hospitalizados en los servicios clínicos de medicina y cirugía de un hospital público.

- Caracterizar a los pacientes hospitalizados en los servicios de medicina y cirugía, según las variables biosociodemográficas.

- Identificar las variables asociadas a la hospitalización que influyen en la percepción de violencia de los pacientes hospital izados en estos servicios clínicos, tales como tiempo de hospitalización, tipo de tratamiento, hospitalizaciones previas, y ayuda requerida.

- Identificar las variables asociadas a la percepción de violencia, tales como: el tipo de conductas violentas percibidas, los horarios, las situaciones, las intensidades en las que sepresentan, las respuestas de afrontamiento, los agentes agresores y los factores que predisponen a estos hechos.

- Identificar la evaluación de la atención re cibida por los pacientes hospitalizados en ambos servicios clínicos. 
- Relacionar las variables biosociodemográficas con las demás variables en estudio.

- Comparar los diversos hallazgos encontrados en los pacientes del servicio de medicina con los encontrados en los pacientes del servicio de cirugía.

\section{Hipótesis de trabajo}

$\mathrm{H}$ 1: Existen diferencias porcentuales significativas en la percepción de violencia de los pacientes hospitalizados en medicina, respecto a los pacientes hospitalizadosen el servicio decirugía de un hospital público.

H2: Existen diferencias porcentuales significativas entre las intensidades de la violencia identificadas hacia otros pacientes hospitalizados en el servicio medicina, respecto la intensidad de la violencia identificada hacia otros pacientes hospitalizados en el servicio de cirugía de un hospital público.

H 3: Existen diferencias porcentuales significativas entre los agentes agresores dispensadores del cuidado identificados por los pacientes hospitalizados en medicina, respecto a los identificados por los pacientes hospitalizados en el servicio de cirugía de un hospital público.

$\mathrm{H}$ 4: Existen diferencias porcentuales significativas entre las situaciones en las que se presentan las conductas violentas percibidas por los pacientes en medicina, respecto a las percibidas por los pacientes hospitalizadosen el servicio de cirugía de un hospital público.

\section{MARCO DE REFERENCIA}

Frente al escenario de ascenso de la violencia y sus consecuencias en la sociedad, se ha tratado de identificar, explicar y comprender cómo la violencia surge en los diferentes ambientes en el que las relaciones humanas se presentan, cómo las personas son potenciales víctimas o victimarios de estos hechos. En virtud de la multicausalidad reconocida de este fenómeno deviolencia, Chapell y Di M artino (1998), basados en el modelo de Payner y Warner, presentan el denominado Modelo Interactivo en el que identifican los factores de riesgo tanto individuales como sociales, que determinan la manifestación de conductas violentas en el ámbito del trabajo, que es aplicable también a otras dimensiones del quehacer humano y que fue consi derado para la presente investigación, aplicando el modelo al ambiente hospitalario (Figura N1).

O tros autores también establecen que no existe un solo factor que dé cuenta adecuadamentedelos niveles de violencia y que, al discutir sobrelosfactoresque contribuyen o que inhiben al comportamiento violento, es decir, al considerar factores de riesgo y de protección, es necesario distinguir entre los que operan a nivel individual, del ambiente (por ejemplo: el hogar) y a nivel comunitario o de la sociedad. Es importante entonces tener en cuenta los antecedentes de violencia social y situacional, aquellas características del ambiente físico y social que pueden motivar 0 inhibir directa o indirectamente los comportamientos violentos entre los individuos (Buinic y cols., 1999; OM S, 2002). De este modo la violencia se constituye hoy en un tema relevante de investigación. 
Figura No1. Violencia laboral: Un Modelo Interactivo de Chapell y Di Martino (1998) aplicado al ambiente intrahospitalario.

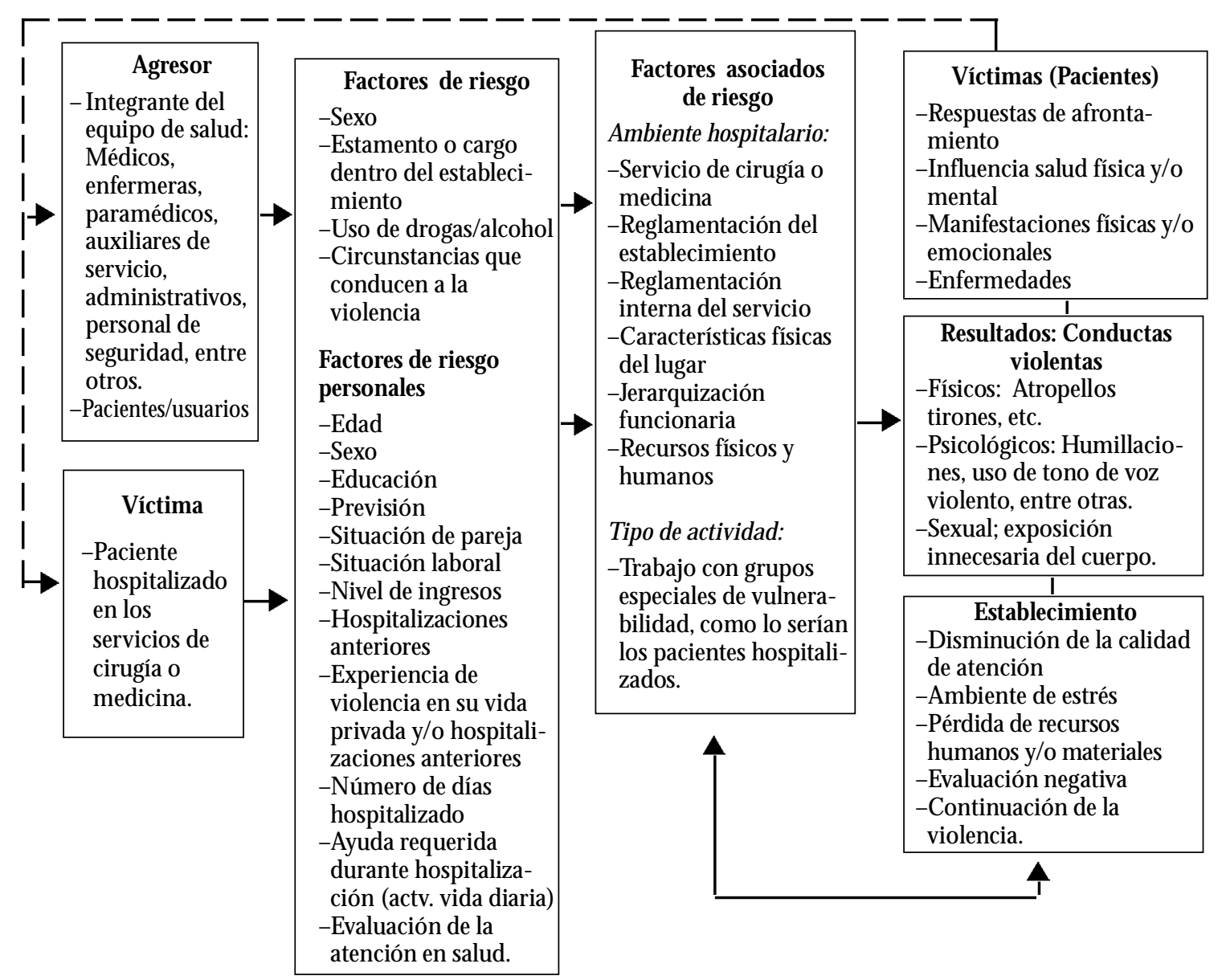

Fuente: “Percepción deviolencia delos pacientes hospitalizadosen losservicios demedicinay cirugía deun hospital público. Octava Región”. Burgos, M y Paravic, T.

Diversos autores identifican aspectos 0 preocupaciones queaparecen esenciales en los pacientes expuestos a alguna enfermedad, entre éstos se encuentran: la consideración por sus valores, adecuada comunicación y educación, respeto y consideración, cuidados integrados, apoyo emocional, alivio de miedos, ansiedades (Delblanco, 1992), trato amable, un clima de confianza y adecuada disposición (Sáez, 1995). Se reconocen además aquellos atributos negativos que son identificados en el personal de salud, tales como: agresividad y mal carácter, descuido, indiferencia y falta de compromiso (M anccini, 1992).

Para Ferreira (1997), el usuario adopta el "rol de paciente" aceptando pasivamente la atención, lo que podría conducir a no manifestar su desagrado a ciertas actitudes y comportamientos, por miedo a represalias posteriores, lo que contribuiría considerablemente 
a la no identificación de actos violentos de parte de los prestadores durante la atención que recibe.

Campos (1997) y Costa (1996), en Brasil, concluyen que existen importantes diferencias en el trato y por ende en el respeto a los derechos de los pacientes según la clase social a la que éstos pertenecen, favoreciendo aque llos que se encuentran en mejores estratos socioeconómicos, presentándose esta situación comúnmenteen los sistemas deatención pública de salud. Estehallazgo es compartido en un trabajo realizado también en Brasil por Pereira (2000), el que concluye que existe relación estrecha entrefactores dependientes del paciente, los quedeterminan la atención y trato brindado de parte de los funcionarios en los servicios públicos de salud, entre los que se encuentran: la pobreza y bajo nivel educacional. Las personas que responden a esteperfil, señala el autor, no poseen un capital lingüístico paran egociar con los profesionales de salud y exigir una buena atención en salud.

La relación entre los prestadores de atención y el pacientereceptor de los serviciosinfluyeen estosúltimos. Este aspecto se ha considerado como el área queprovoca más estrés en los pacientes hospitalizados, especialmente en la interacción que se gesta con el médico y las enfermeras. Esta es la conclusión de un estudio aplicado en un hospital chileno a pacientes previos a ser intervenidos quirúrgicamente, en los que la indiferencia en el trato y la falta de atención representaron factores provocadores de altos niveles de estrés en los pacientes (Roa, 1995). En esta misma investigación la satisfacción de necesidades de eliminación, vesical e intestinal, eran factores que representaban alto grado de estrés en los pacientes al tener que depender de los funcionarios de salud para satisfacerlas adecuadamente.

López (1994), en un estudio detipo exploratorio descriptivo sobre la percepción de la calidad de atención en salud de un grupo de mujeres puérperas de sectores populares chilenos, que participaban en un proyecto desa- lud post-parto y promoción de lactancia materna, encontraron que la evaluación que hicieron las encuestadas respecto a la atención recibida presentaba un cuestionamiento claro al concepto de atención en salud y permitió identificar algunas prácticas que podrían ser consideradas como violencia institucional, según concluyen los autores.

Otros estudios encontrados abordan la dimensión y características de las relaciones establecidas entre prestadores y usuarios de los servicios, pero enfocados en la temática de satisfacción usuaria y evaluación de la atención en salud, que de una forma indirecta se relacionan muchas veces con el fenómeno en estudio, pero que no se detallarán en el presente artículo.

\section{MATERIALESY MÉTODOS}

Tipo de estudio: La presente investigación corresponde a un estudio de abordaje cuantitativo de tipo descriptivo, transversal, correlacional y comparativo, queabordael fenómeno en el lugar de su ocurrencia.

Universo: Estuvo constituido por todos los pacientes hospitalizados dados de alta en los servicios clínicos de medicina y cirugía de un hospital público, duranteel período comprendido entre los meses de mayo a julio del año 2002, los que correspondieron a 700 pacientes del servicio de medicina y 980 de cirugía, haciendo un total de 1.680 pacientes.

Muestra: Estuvo conformada por 70 pacientes del servicio de medicina y 104 pacientes del servicio de cirugía, elegidos al azar de la lista de egreso diaria.

Instrumento recolector de datos: Para la obtención de la información se aplicó la encuesta "Percepción de violencia en los contextos de salud", elaborada por Paravic y cols. (2000), la que fue adaptada al contexto hospitalario y aplicada por las autoras. La recolección de los 
datos fue realizada mediante una entrevista, en un lugar implementado para ello, en el recinto hospitalario.

Validez: Se cauteló la validez a través de grupos focales y el análisis de expertos pertenecientes a las áreas de sociología, psicología y de enfermería de la Universidad de Concepción. Posteriormente fue aplicada una prueba piloto.

Confiabilidad: Fue obtenida con el coeficiente de correlación alpha de Cronbach, el que arrojó un valor de 0,81.

Procesamiento delos datos: Los resultados ob- tenidos fueron procesados mediante los programas estadísticos: SAS, SPSS 8,0 y Statistica.

Aspectos éticos: Para realizar la investigación se contó con la autorización de los directivos del hospital, y el consentimiento informado de cada paciente participante, asegurando el anonimato y la confidencialidad de los datos otorgados.

\section{RESULTADOS}

Algunas de las variables biosociodemográficas resultantes son presentadas a través de las siguientes gráficas.

\begin{tabular}{|l|l|}
\hline & Medicina \\
\hline
\end{tabular}

Gráfico N 01 . Distribución de pacientes según servicio clínico de hospitalización.

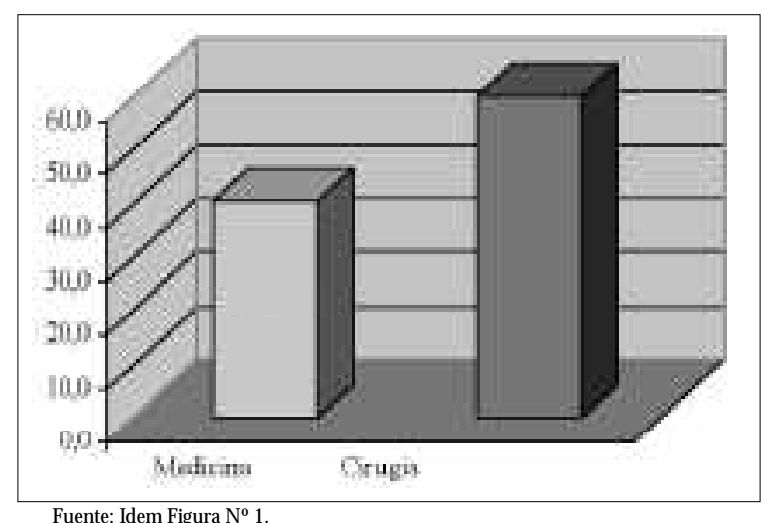

Gráfico No3. Distribución de pacientes hospitalizados según grupo etáreo.

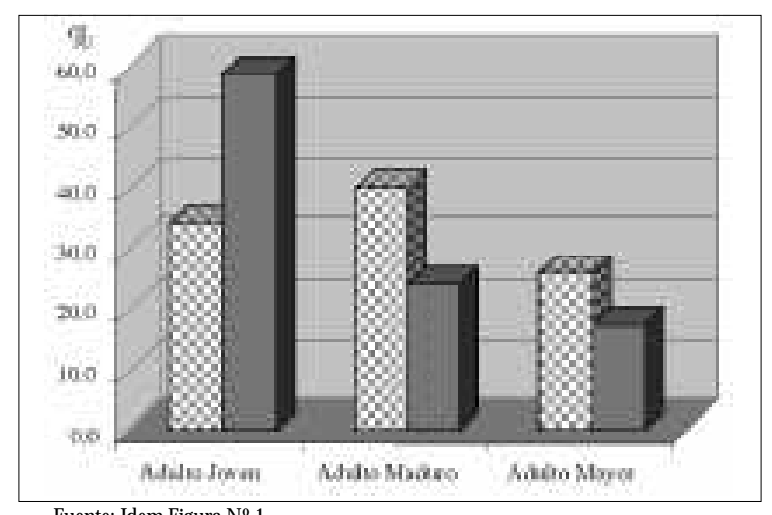

Gráfico № 2. Distribución de pacientes hospitalizados según sexo.

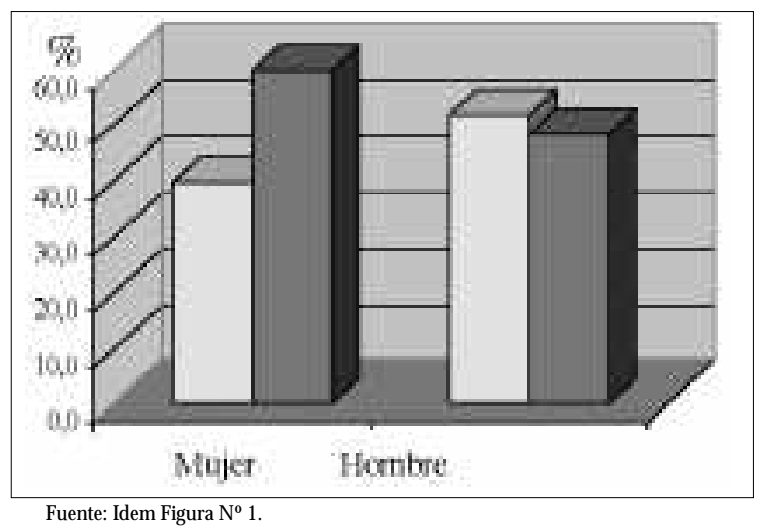

Gráfico № 4. Distribución de pacientes hospitalizados según nivel de educación.

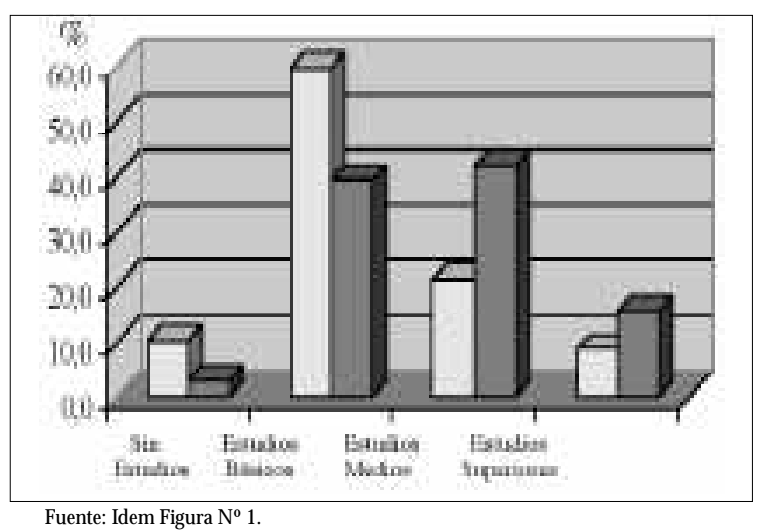


Gráfico Nㅇ․ Percepción de violencia de los pacientes hospitalizados en medicina y cirugía.

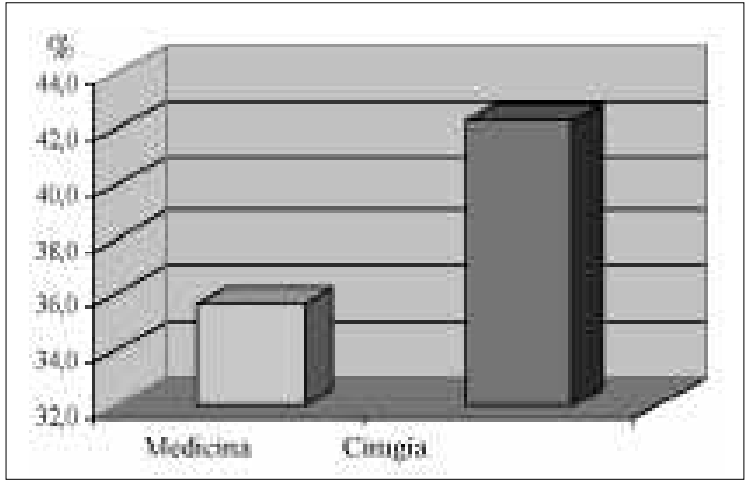

Fuente: Idem Figura № 1 .

Gráfico № 7. Tipos de violencia percibidas por los pacientes en medicina y cirugía.

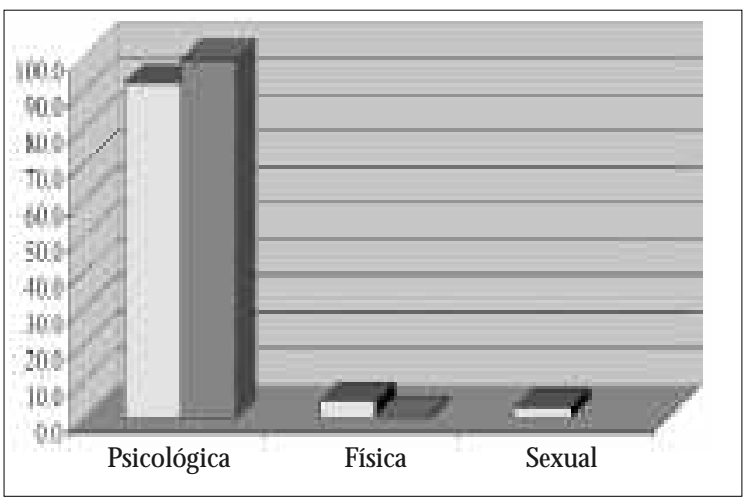

Fuente: Idem Figura № 1 .

Gráfico N ㅇ. 9. Agentes agresores más frecuentes identificados.

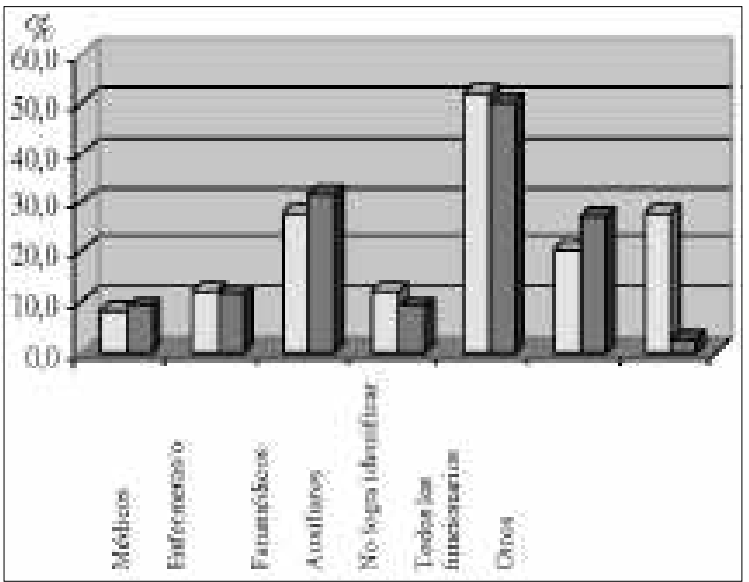

Fuente: Idem Figura № 1.
Gráfico N ㅇ. - Intensidad delas conductas violentas percibidas por los pacientes en medicina y cirugía.

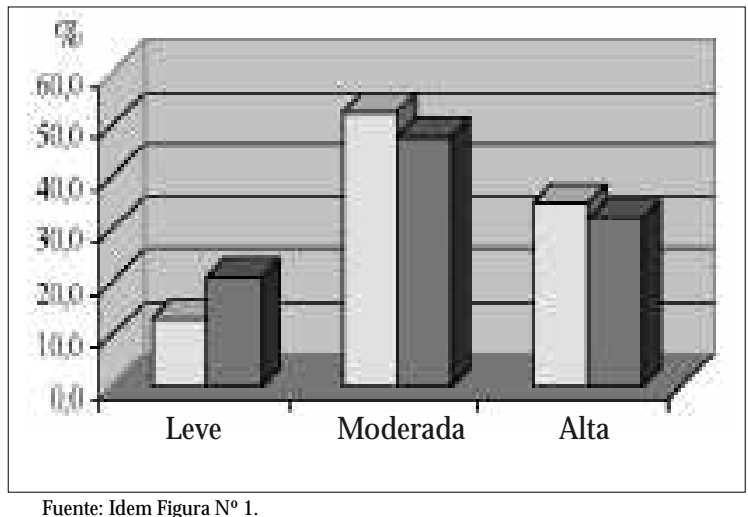

Gráfico No 8. Conductas de violencia psicológica percibidas con más frecuencia por los pacientes de Medicina y Cirugía.

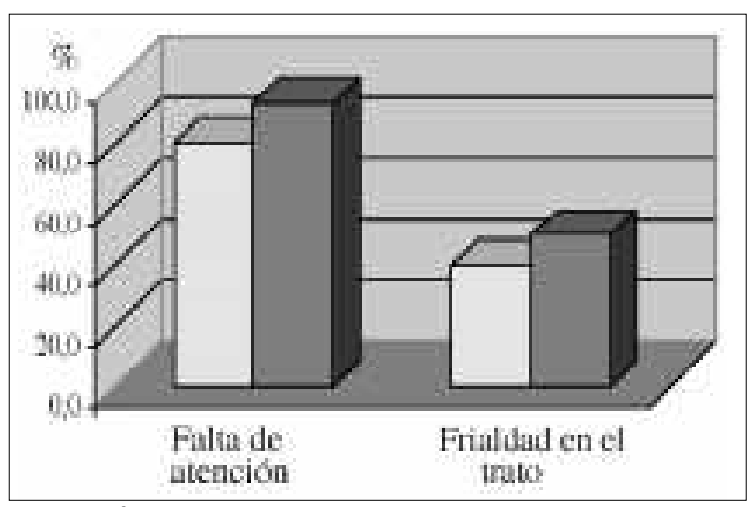

Fuente: Idem Figura № 1.

Gráfico № 10. Situaciones en las que se presentaron las conductas violentas.

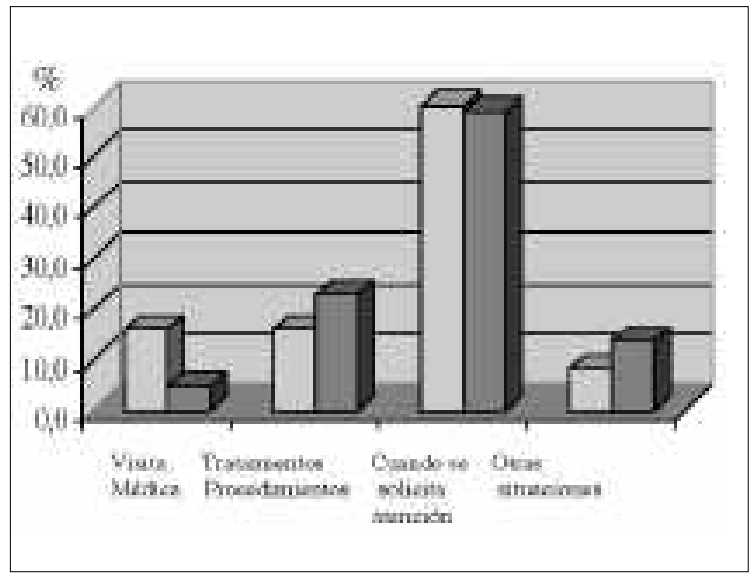

Fuente: Idem Figura № 1 . 
Gráfico $\mathrm{N}$ 11. Horario en el que se presentan las conductas violentas.

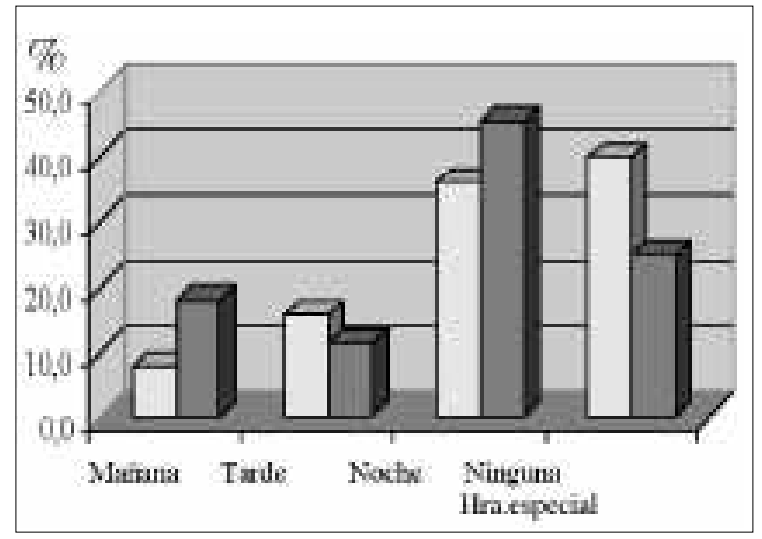

Fuente: Idem Figura № 1.

Gráfico No 13. Respuestas de afrontamiento de los pacientes frente a las conductas violentas percibidas.

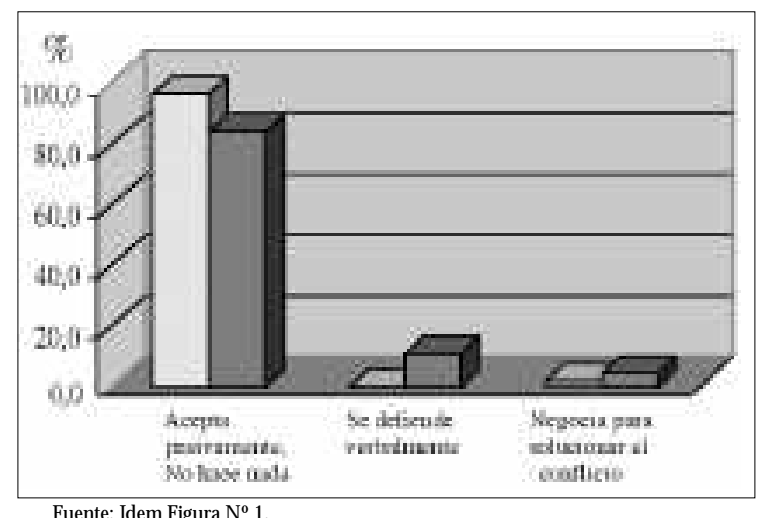

Al realizar el análisis de regresión logística, el modelo óptimo que contempló aqueIlas variables con mayor asociación estadística con la percepción de violencia -y por lo tanto consideradas predictoras de ésta- fueron: edad, nivel de educación y la evaluación dela atención de salud. De este modo los pacientes más jóvenes, con mayor nivel educacional y que no evaluaron de la mejor forma la atención durantela hospitalización, fueron quienes percibieron violencia.
Gráfico № 12. Factores predisponentes a conductas violentas.

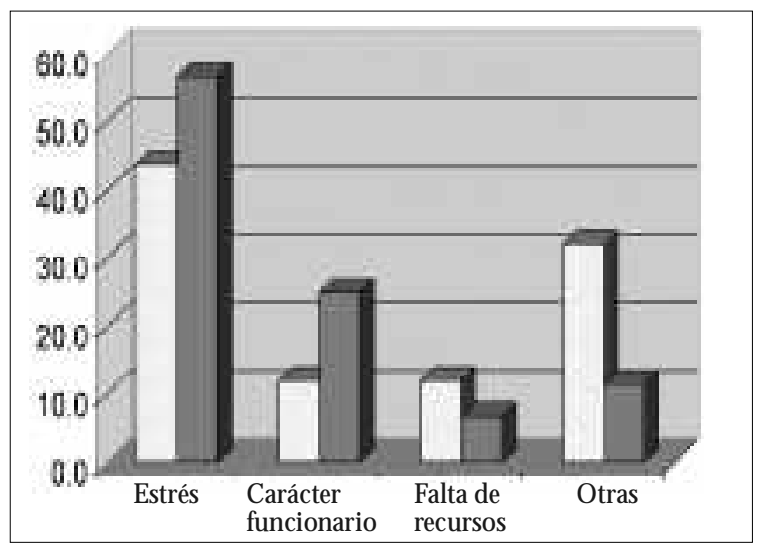

Fuente: Idem Figura № 1.

Gráfico № 14. Evaluación de la atención de salud durante la hospitalización.

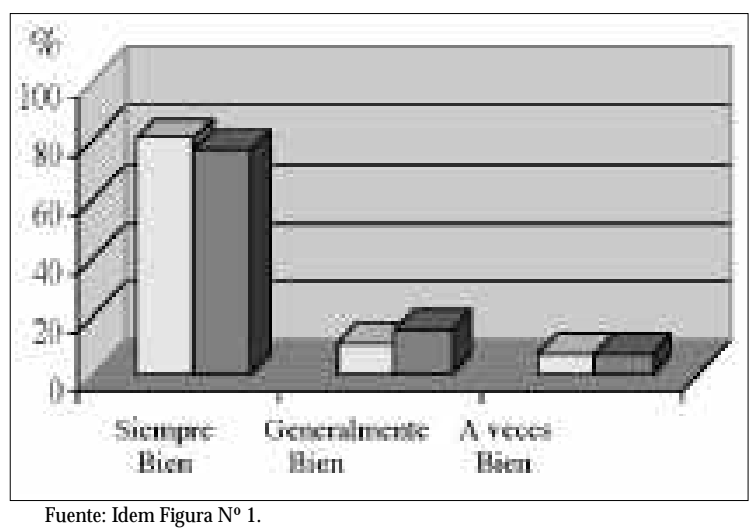

\section{DISCUSIÓN}

La percepción de violencia identificada en un contexto hospitalario representa un hallazgo considerado importante, puesto que esto significa que no solamente los trabajadores de salud son víctimas de violencia (CIE, 1999; Sánchez, 2002), sino que esta problemática afecta también a los usuarios de los servicios de hospitalización. Este hecho debe llamar a la reflexión sobre la forma en la que se está 
llevando a cabo la atención a los pacientes, queson nuestro principal sujeto deatención, y cómo se vulneran sus derechos.

Los pacientesidentificaron una o más conductas violentas, las que correspondieron en su gran mayoría a manifestaciones de violencia de tipo psicológico. Dentro de las que se destacaban, la "falta de atención" en la satisfacción denecesidades deaseo y/o confort fue ejemplificada recurrentemente por los pacientes en estudio con el tiempo prolongado que demoraba el personal de salud en atender llamados de solicitud y retiro de chatas, y la falta de cooperación en la realización del aseo personal, en especial de aquellos más dependientes. Esto se puede relacionar con la escasez de personal para la atención específica de aseo y confort delos pacientes hospitalizados; el recurso humano disponible es limitado, y no logra atender a tiempo a todos los llamados de los usuarios que requieren atención.

La "frialdad en el trato" del personal de salud es otro aspecto considerado como violentador por el paciente/usuario de los servicioshospitalarios, quese identifican con conductas poco empáticas e impersonales. Los pacientes identifican este trato impersonal, por ejemplo, al ser tuteados por el personal queles atiende, y a la relación indiferente que losfuncionarios de salud establecen con ellos. Estetrato frío eindiferentetambién esun factor estresanteen los pacientes durantela hospitalización, ya que las personas en esta condición necesitan establecer una relación de confianza con el personal a cargo de su cuidado (Roa, 1995). A este punto se refiere también Rodríguez (1999), cuando dice que la calidez en la atención queotorga el equipo de salud se torna un aspecto importante -el saber escuchar, establecer una dinámica de relación que puede ir desde el contacto visual hasta el abrazo estrecho, sonreír y el consolar sinceramente- que condiciona la relación entre prestadores y usuarios. Las investigadoras comparten ampliamentela necesidad que existe de establecer una comunicación basada en la empatía y respeto con el paciente; sin embargo, esta relación se ve alterada tal vez, por un lado, con la demanda asistencial -que muchas veces limita la comunicación más estrecha con los pacientes- y, por otro, por las conductas de algunos funcionarios que aún consideran al paciente como un mero receptor de cuidados, y no como un integrante activo de la atención en salud.

El queel mayor porcentajede pacientes no logre diferenciar claramente al estamento de los funcionarios agresores, puede estar relacionado con el hecho de que cuando un paciente es ingresado a un hospital es separado de su ambientefamiliar - conocido y establey sometido a otro extraño, en el quedeberelacionarsecon múltiples personas, sufriendo algún grado de confusión, especialmente cuando el personal de salud no se identifica previamente o no porta una identificación clara y/o por lo similar del vestuario. En este punto es necesario destacar que especial confusión se presentaba en los casos en que los pacientes debían diferenciar las enfermeras de los paramédicos, quedando demanifiesto la poca distinción querealiza el usuario del profesional universitario y del técnico, identificándolos a ambos como enfermeras. Por otro lado, esto puede estar asociado además a quelos pacientes entrevistados no quieran señalar un estamento determinado, por no comprometerse mayormente en la denuncia de un hecho de violencia identificando al agente agresor.

Los pacientes hospitalizados señalaron en el presente estudio que en el turno de noche es cuando se presenta la mayor cantidad de conductas violentas. El turno denochecorresponde a la jornada de trabajo intrahospitalaria en que existe menor cantidad de funcionarios, comparados con los turnos diurnos. Durante el día son múltiples personas las que encuentran en los servicios clínicosatendiendo y satisfaciendo las necesidades de los pacientes, no solamente se encuentra el personal hospitalario (enfermeras, técnicos paramédicos, médicos, auxiliares deservicio), sino queademás se están: alumnos deenfermería, medicina, técnicos paramédicos y los fami- 
liares en las horas devisita. Todas estas personas colaboran durante el día con las diversas actividades asistenciales. Sin embargo, durante la noche sólo se encuentran, y en menor número, losfuncionarios del hospital, losque, aunque realizan menor cantidad de actividades que en el turno diurno, deben también realizar controles, procedimientos y diversos cuidados a los pacientes hospitalizados. ¿Será queel personal del turno nocturno es insuficiente o que la atención brindada en este horario difiere del resto?

Chapell y Di Martino (1998) reconocen que el estrés es un factor determinante en la presentación de comportamientos violentos en los individuos. Este aspecto ha sido también analizado por el CIE (2001), el queseñala que una de las causas de estrés en el personal de salud es el tratar con la muerte y los moribundos, conflictos entre compañeros de trabajo, preparación inadecuada para tratar necesidades emocionales de pacientes y sus familias, y precisamente la sobrecarga o exceso de trabajo, la quea su vez puedellevar a la falta de atención en las actividades y al cambio de comportamientos. Los mismos profesionales de enfermería, según Sánchez (2002), atribuyen ala presión asistencial como el principal factor asociado a la violencia. Este aspecto requiere mayor atención, ya que tanto los dispensadores de los cuidados de salud como los propios pacientes receptores de los cuidados, reconocen al estrés como causal de violencia.

La mayoría de los pacientes que señalan percibir conductas violentas durante la atención recibida en la hospitalización, aceptan estas situaciones sin decir ni hacer nada. El adoptar unaactitud pasivafrentea las conductas violentas puede relacionarse con la relación asimétrica y jerárquica que se establece entre el personal de salud y los pacientes en los hospitales públicos (Rocha y cols., 2000), en la que los pacientes en una situación de dependencia en mayor o menor grado toleran las diversas conductas y manifestaciones quesurgen durantelahospitalización. Ferreira y Figueiredo (1997), en un estudio basado en las relaciones de poder entre las enfermeras y pacientes adultos hospitalizados, estosúltimos adoptaban una actitud sumisa, pasiva y obediente, aceptando la atención de salud desde su rol pasivo, como mero receptor de los cuidados, actitud que se ha tratado de cambiar impulsando la participación activa de los usuarios en la atención en salud, por ejemplo a través de la divulgación de los deberes y derechos del paciente.

Aunque desde hace un tiempo el tema de los derechos de los pacientes en nuestro país se ha difundido, especialmentea través delos medios decomunicación, los usuarios del sistema público aún mantienen un "rol de paciente", presentando una actitud pasiva, impulsada por el propio personal de salud, que limita su expresión o juicios respecto a la atención recibida. Según la experiencia de las investigadoras, esta situación se presenta especialmente en los pacientes de estratos educacionales y económicos más bajos, los que además "por la gratuidad de la atención recibida" suelen conformarse con las prestaciones sin enjuiciar actos que pudieran parecerles cuestionables, como son los comportamientos violentos. I gual experiencia refierePereira (2000) en trabajos realizados en Brasil.

Con el tiempo lo más probable, como ya se está presentando, la actitud y exigencias de los pacientes respecto a la atención en salud que recibe irán en aumento. Goic (2000) señala que esto se debe a que, por un lado, existe mayor difusión e información del común de las personas respecto a sus derechos en salud, como ocurre en países desarrollados, y, por otro, han aumentado las expectativas de las personas en salud, lo quese estátraduciendo en instrumentos legales que obligan a los prestadores de los servicios sanitarios, autoridades de salud y a los gobiernos, a un esfuerzo por no solamente satisfacer las necesidades en salud, sino que se traduce además en un trato adecuado al paciente.

A pesar dehaber percibido conductas violentas durantela hospitalización, muchospa- 
cientes manifestaron quesiemprehabían sido bien atendidos durantela hospitalización. Esta situación puede estar asociada a que los pacientes en el momento del alta hospitalaria consideran la atención en general, estimando su recuperación y egreso hospitalario como un éxito, pasando a un segundo plano cualquier evento desagradable experimentado durante la atención, como lo serían las conductas violentas. Por otro lado, las personas tienden a dar respuestas que sean socialmente aceptadas y además se puede interpretar como una evidencia más del temor de enjuiciar la atención de salud.

Según los autores M oris, Riverosy Pucheau (1996) y las autoras de este estudio, muchos delos pacientes manifestaron temor a los juicios devaloración de la atención recibida, especialmente en los servicios públicos de salud, ya que saben que en al gún momento de sus vidas volverán a estos servicios y temen las represalias de los funcionarios si es que ellos evalúan negativamente la atención. Los pacientes no permitieron que esto quedase consignado en el registro escrito, siendo por lo tanto el porcentaje real de percepción de violencia mayor a lo que se expone en los resultados.

\section{CONCLUSIONESY SUGERENCIAS}

- Un alto porcentaje de pacientes durante la hospitalización perciben conductas que catalogan como violentas de parte de los funcionarios que les atienden, siendo este porcentaje en la realidad probablemente mayor, ya que algunos pacientes no quisieron reconocer violencia durante la atención por temor a represalias posteriores.

- La mayoría de los pacientes hospitalizados que percibe violencia durante la atención refiere que ésta fue de una intensidad moderada y corresponden a manifestaciones del tipo de violencia psicológica en la gran mayoría de los casos, dentro de las que se destacala "falta deatención" y el "trato frío".
- Un alto porcentaje de pacientes reconoceno diferenciar al agente agresor hospitalario.

- Los pacientes identificaron que las conductas violentas se presentaban mayoritariamente cuando solicitaban atención y en el horario de turno de noche.

- El factor predisponente del agresor para efectuar conductas violentas, según la percepción de los pacientes corresponde al estrés por sobrecarga de trabajo.

- El mayor porcentaje de pacientes frente a las conductas violentas adoptaba una actitud pasiva.

- La evaluación de la atención recibida durantela hospitalización, realizada por el mayor porcentaje de pacientes que conformaron la muestra, refirieron haber sido siempre bien atendidos a pesar quepercibieron conductas violentas, aceptando éstas durantela hospitalización.

- Mediante el análisis de regresión logística, independiente del servicio clínico de procedencia, los pacientes másjóvenes, con mayor nivel educacional y quetienen una mala evaluación de la atención de salud, son los que mayormente perciben violencia en el contexto intra-hospitalario.

Según los resultados obtenidos, se hace necesario implementar medidas orientadas a la disminución y manejo adecuado del estrés por sobrecarga de trabajo del personal de salud, reconocido como el principal factor predisponente a la violencia, a través del aumento defuncionarios, especialmentedurante los turnos nocturnos. Además, de ejecutar programas destinadosa promover en losfuncionarios los derechos y deberes delos pacientes, enfatizando en la relación de ayuda efectiva que se debe establecer con los usuarios.

Se hace imperativo reconocer la diferencia entre los estamentosfuncionarios desalud de partedelos pacientes, promoviendo el uso de una identificación clara y visible, para delimitar responsabilidades cuando así se requiera.

Las futuras investigaciones en esta línea deben cautelar el momento en el que serán 
recogidos los datos, si bien para esta investigación fue considerado el momento del alta hospitalaria, el hecho de tener que volver a controles posteriores y la posibilidad de un eventual reingreso al servicio clínico, no liberaba totalmente a los pacientes a manifestar sus percepciones sin temor.

Este hallazgo de percepción de violencia, en un medio cuyo objetivo es atender las necesidades de salud de las personas, debe llevar a una profunda reflexión de cómo se están realizando las prestaciones sanitarias, la que ciertamente debe contemplar la forma cómo nosacercamos y presentamosal paciente, losgestos, tono devoz, uso de palabras claras basadas en un diálogo respetuoso y empático, nunca olvidar que el que está al otro lado es un ser humano como cualquier otro, que merece un trato digno.

\section{REFERENCIAS BIBLIOGRÁFICAS}

BUINIC, M .; M orrison, A y Shifter, M . (1999). La violencia en América Latina y el Caribe: Un marco de referencia para la acción. Extraído en octubre 15, disponibleen http://www.iadb.org/sds/publication/ publication_515_s.htm

CAM POS (1997). Eñ: Proyecto de un estudo diagnostico das condiçoes de atendimento aos direitos da pessoa internada em um serviço público hospitalar na cidade de Cuiaba-M G. Brazil.

CHAPELL, D.y Di Martino, V. (1998). ViolenceatWork. Génova: International Labour Office.

CON SEJO Internacional de Enfermería (1999a). La violencia. Epidemia mundial. Página Informativa del CIE. Extraído el 11 septiembre, 2000, disponible en http://www.icn.ch/matters_violencesp.htm

CONSEJO Internacional deEnfermería (1999b). El incremento de la violencia en el lugar de trabajo es una amenaza para la enfermería y la prestación de los cuidados de salud. En Comunicado de prensa CIE. Extraído el 28 julio 2002, disponible en http:/ /www.icn.ch/prviolence 99sp.htm

CONSEJO Internacional de Énfermería(2001). EI CIE aborda el estrés en el trabajo y la amenaza que supone para la salud del trabajador. Boletín informativo, La enfermería importa. Extraído el 06 octubre 2001, disponible en http://www.icn.ch/ matters_stressp.htm

CONSEJO Internacional de Enfermería (2002). Nue- vos estudios muestran que la violencia en el lugar de trabajo es una amenaza para los servicios de salud en todo el mundo. Comunicado de prensa CIE. Extraído el 26 septiembre2002, disponible en http:/ /www.icn.ch/violencia/pr10_02sp.htm

COSTA (1996). En: Rocha, W.; Rochti, W. y Bellato, R. Proyecto de un estudo diagnostico das condiçoes de atendimento aos direitos da pessoa internada em um serviço público hospitalar na cidade de Cuiaba-M G. Brazil.

DELBLAN CO, T. (1992). Enriching the doctor-patient relationship by inviting the patient's perspective. Annals of Internal Medicine, 116, 414-418.

FERREIRA, M . y Figueiredo, N. (1997). Os mecanismos disciplinadores do hospital: As(os) enfermeiras (os) eo poder sobre o corpo de clientehospitalizado. Extraído el 08 noviembre 2002, disponible en http:// bases.bireme.br

GOIC, A. (2000). Es hora depensar en los derechos de los pacientes. Unaintroducción. Revista M édicade Chile, 128, 1371-1373.

ICETA, M . (1996). Bioética ipara qué? RevistaBioética y Ciencias de la Salud, 2 (2), 39-34.

JIMÉNEZ, P. (2000). Cuidados de la autoestima del paciente hospitalizado. Revista Sociedad de Enfermería Geriátrica y Gerontológica, 2, 71-78.

JIM ÉN EZ, J. H.; Coeymans, M . y Gili, M . (1990). Percepción de calidad de la atención médica por los usuarios. En: Eficiencia y calidad del sistema desalud en Chile. Corporación de promoción Universitaria (CPU), pp. 265-303.

KINGM A, M . (1999). El incremento dela violencia en el lugar de trabajo es una amenaza para la enfermería y la prestación de los cuidados de salud. En Comunicado de prensa CIE. Extraído el 28 julio 2001, disponibleen http://www.icn.ch/prviolence 99 sp.htm

LÓPEZ, S. (1994). Percepción dela calidad deatención en salud de un grupo de mujeres de sectores populares. Extraído el 10 octubre 2002, disponible en http://localhost:81/cgi-bin/wxislind.exe/iah/cdrom/

MARTíNEZ, A. (1997). El profesional de enfermería en su entorno de trabajo. Humanización dela asistencia. En L. Pastor y F. León (Comp.), M anual de ética y legislación en enfermería, Madrid: M osby/ Doyma, pp. 171-176.

M ANCCINI, R.; Uribe, R., Sepúlveda, E. y Robles, M . (1992). Imagen de los médicos. Rev. Vida M édica, 44,16-22.

M ORIS, I.; Riveros, N. y Pucheau, J. (1996). Percepción de los usuarios que ingresan al servicio de cirugía del hospital Herminda Martin de Chillán. Revista H orizonte de Enfermería, 7(1), 36-42.

MORO, A. (1999). El enfermo. En: G. Comolli y I. M ontecelli (Comp.), M anuale di Pastorale Sanitaria. Roma: Camilliane, pp. 211-212. 
ORGANIZACIÓN M undial delaSalud (OMS). (2002). World report on violenceand health. Génova: OMS, pp. 9-11.

PARAVIC, T., Sánchez R. y Valenzuela, S. (2000). Percepción de violencia en los contextos de salud. I Instrumento de Medición/. Universidad de Concepción.

PEREIRA (2000). En: Rocha, W.; Rochti, W. y Bellato, R. Proyecto deun estudo diagnostico das condiçoes de atendimento aos direitos da pessoa internada em um serviço público hospitalar na cidade de Cuiaba-M G. Brazil.

ROA, B. (1995). Factores estresantes percibidos por el paciente en el período pre-operatorio: servicio de cirugía, Hospital Las Higueras de Talcahuano. Tesis para optar al grado de M agister en Enfermería, Departamento deEnfermería, Universidad deConcepción, Concepción, Chile.

ROCH A, W.; Rochti, W. y Bellato, R. (2000). En: Proyecto de un estudo diagnostico das condiçoes de atendimento aos direitos da pessoa internada em um serviço público hospitalar na cidade deCuiabaMG. Brazil.
RODRÍGUEZ, C. (1999). Calidad de la atención en salud y Ley 100 de seguridad social. En: Bioética y Justicia Sanitaria. Colombia: El Bosque, pp. 147182.

SÁEZ, M . (1995). Evaluación de los usuarios delos sistemas deatención desalud. Rev. Vida M édica, 47(1), 51-56.

SÁNCHEZ, R. (2002). Percepción de violencia que afecta a los profesional es de enfermería de un hospital de la O ctava Región. Tesis para optar al grado de Magister en Enfermería, Departamento de Enfermería, Universidad deConcepción, Concepción, Chile.

VACAREZZA, R. (2000). Delos derechos del paciente. Revista M édica de Chile, 128, 1.380-1.384.

VALENZUELA, S. (1995). Factores que influyen en la actitud de los estudiantes de enfermería hacia el cuidado de pacientes durante sus experiencias clínicas médico-quirúrgica. Tesis para optar al grado de Magister en Enfermería, Departamento de Enfermería, Universidad de Concepción, Concepción, Chile. 